\title{
Evaluation Of Findings On Imaging Of Brain In Children With First Recognized Episode Of Fits-Experience At Tertiary Care Hospital Of Quetta
}

\author{
Pari Gul, Ameet Jesrani, Palwasha Gul, Nida Amin Khan
}

ABSTRACT

Objective: To evaluate various causes of fits in children on MRI presenting in Tertiary Care Hospital, Quetta.

Study Design and Setting: A Cross sectional study was conducted in the department of Radiology, Bolan Medical Complex Hospital Quetta from October 2017 to March 2018.

Methodology: A total of 100 children aged 06 months to 13 years were included in the study who presented with seizures to emergency department and neuro clinics. Information obtained from history, clinical examination and MRI brains were recorded. The data was analyzed in SPSS 20.

Results: Among the total 100 children, MRI examination was unremarkable in $45 \%(n=45)$. Neoplastic lesions were the second most common abnormal MRI finding and constituted $10 \%(\mathrm{n}=10)$. Perinatal ischemia and Periventricular leucomalacia were recorded in 9\% $(n=9)$. Congenital aqueductal stenosis in 9\% $(n=9)$, along with Encephalitis/Meningitis also in 8\% $(n=8)$. Brain atrophy was noted in $6 \%(n=6)$. Three cases each of Vascular and Post traumatic changes/gliosis $(n=6,6 \%)$ and one case of each of Hydrocephalus/Aqueductal stenosis, Infarct, Malformation of cortical development, Leucodystrophy, Agenesis of corpus callosum, Arachnoid cyst and Hydatid cyst ( $\mathrm{n}=7,7 \%)$

Conclusion: Brain magnetic resonance imaging was successful in detecting structural abnormalities and it can be trusted to detect seizure foci in pediatric patients.

Keywords: Brain. Children. Fits, MRI.

\section{INTRODUCTION:}

Epilepsy is one of the most frequently seen neurologic disorders within childhood. International League against Epilepsy defines epilepsy as; at least two unprovoked or reflex seizures $>24 \mathrm{~h}$ apart. Epilepsy affects 50 million people around the world and half of them start in childhood period..$^{1-3}$

The overall prevalence of epilepsy in Pakistan is 9.99 per 1000 population. ${ }^{4}$ Highest prevalence is seen in people younger than 30 years of age. Higher prevalence is observed in rural population. Aetiology of epilepsy is more commonly identified in paediatric population. ${ }^{4}$

A seizure is a sudden, uncontrolled electrical disturbance in the brain. It is a symptom that points towards an underlying neurological disease or epilepsy. $1 \%$ of all emergency

Г

I Assistant Professor, Department of Radiology,

I Bolan Medical Complex Hospital, Quetta

I Ameet Jesrani

Assistant Professor, Department of Radiology,

Sindh Institute of Urology and Transplantation, Karachi

I E-mail: ameet.jesrani@yahoo.com

I

Palwasha Gul

Senior Registrar, Department of Radiology,

I Bolan Medical Complex Hospital, Quetta

I Nida Amin Khan

I Resident, Department of Radiology,

Sindh Institute of Urology and Transplantation, Karachi

I Received: 22-10-2018

Accepted: 02-05-2019 department visits are seizure related symptoms with a higher incidents being of infants and children. ${ }^{5}$ Anatomic classifications define seizures of two types. One that has its focus of origin in temporal lobe and others that arise from a focus other than temporal lobe. ${ }^{6}$ The most advance classification is based on the episode as described by the patient or the observer. There are focal seizures that affect a group of muscles or a body part. These may become generalized involving whole body also described as convulsions. Generalized seizures are the ones that involve whole body. ${ }^{7,8}$ In general population, the mean prevalence of epilepsy is established to be $8 \% .{ }^{9}$ Epilepsy is a debilitating disorder that renders a person deprived of their psychosocial and physical standing. The psychosocial aspect of this disease is so enormous that it affects not only the patient but also his/her family. The stigma is so crude that it spans from mental well being to restriction in everyday activities and acceptance in society.

Although there is impressive advancement in the medical treatment of epilepsy, there are still cases that are refractory to treatment. It is worth noting that most of the refractory cases are due to underlying brain disorders that manifest as structural changes. Here, brain imaging plays an important role so as to guide the neurologist if any immediate surgical or medical intervention is needed for the treatment of the patient.

Computed Tomography of head in infants younger than six months presenting in emergency department with a first seizure, demonstrated a brain lesion in $50 \%$ of cases as concluded in a study by Harden et al. ${ }^{10}$ 
King et al. reported abnormal Magnetic Resonance Imaging (MRI) findings when all seizures evaluated as 35 in 263 patients (13.3\%).4 With the increase of availability of high quality MRI; lesions that are not detected in Computed Tomography such as heterotopias and mesial temporal sclerosis that are both associated with childhood onset seizures can be visualised. ${ }^{11-14}$

Magnetic Resonance Imaging is a strong modality in neuroimaging and with its protocols and sequences, has the capability of demonstrating soft tissue and brain lesions with higher precision resulting in renaissance in evaluation and management of epilepsy. ${ }^{15}$

Sequences of MRI brain and their importance in detection of specific disease processes may aid in devising standard protocols for local community in near future.

The aim of our study is to highlight the strength of MRI brain as a diagnostic tool for detection and evaluation of structural abnormalities in pediatric population presenting with seizures and help preventing the possible episodes of seizures in future by treating the cause in affected childrens and let them to have brightness in their life.

\section{METHODOLOGY:}

This cross-sectional study was conducted at the Department of Radiology, Bolan Medical Complex Hospital Quetta from October 2017 to March 2018. During this period, 100 patients aged 6 months to 13 years with a history of first episode of seizures were included and children with a known underlying genetic or metabolic disorder and children with a significant behavioral or psychiatric disorder were excluded. Children with a symptomatic seizure due to intoxication or drug ingestion or a transient electrolyte or metabolic derangement were also excluded from the study. The patients who were too young or irritable were sedated in MRI room by a qualified anesthetist to carry out MRI examination smoothly and optimally. The MRI examination was performed on 0.3 Tesla Hitachi Airis II\%. The standard protocol used included T1, T2 and FLAIR sequences. MRI contrast gadolinium was administered when required as decided by radiologist. The MRI studies were viewed by consultant radiologists of our department. The reports and findings were recorded. The prescribed proforma was filled. The data was analyzed by SPSS- 20 .

\section{RESULTS:}

Among the total 100 children, MRI examination was unremarkable in $45 \%(n=45)$. Neoplastic lesions were the second most common abnormal MRI finding and constituted $10 \%(\mathrm{n}=10)$. Perinatal ischemia and Periventricular leucomalacia were recorded in $9 \%(\mathrm{n}=9)$. Congenital aqueductal stenosis in 9\% $(\mathrm{n}=9)$, and Encephalitis/Meningitis in $8 \%(\mathrm{n}=8)$.

Brain atrophy was noted in $6 \%(\mathrm{n}=6)$. Three cases each of Vascular and Post traumatic changes/gliosis $(n=6,6 \%)$ and

one case of each of Hydrocephalus/Aqueductal stenosis, Infarct, Malformation of cortical development, Leucodystrophy, Agenesis of corpus callosum, Arachnoid cyst and Hydatid cyst ( $\mathrm{n}=7,7 \%, 1$ case each). Percentages and no of patients are shown in Pie Chart. Figure 1 is showing case of Tuberculous Meningitis as it is showing increased enhancement in basilar meninges along with significant enhancement of basal cisterns on postcontrast T1WI. Figure 2 is showing deficient white matter with indentation of lateral ventricles by gyri on T2WI. Figure 3 demonstrates large avidly enhancing mass arising from left lateral ventricle with hydrocephalous on postcontrast T1WI. Figure 4 showing symmetrical abnormal signals in the Periventricular occipitoparietal white matter with band of enhancement at the active edge of the demyelination on postcontrast T1WI.

Figure 1: Imaging In Peadiatric Population

$\square$ Unremarkable
$\square$ Tumor
$\square$ Perinatal Ischemia +
Periventricular Leucomalacia
$\square$ Congenital
$\square$ Encephalitis/Meningitis
$\square$ Brain Atrophy
$\square$ Vascular
$\square$ Post Traumatic changes/gliosis
$\square$ Others

\section{DISCUSSION:}

Epilepsy is a fairly prevalent neurological disorder with a long course , physical and psychological debilitation. There is inadequate literature available regarding epilepsy in pediatric population in Pakistani population .

Seven in every thousand children were found to have epilepsy in the district of Gujranwala of Punjab, according to a study done by Muhammad Akbar Malik and colleagues. ${ }^{16}$

There is shortage of detailed studies of neuroimaging in our population.

Clinicians can revolutionize the evaluation and management of patients in the light of imaging findings.

Our study concluded that $55 \%$ showed structural lesions in brain in accordance with a previous study that showed 51.3 $\%$ of cases having positive MRI finding (Resta et al ). ${ }^{16}$

Another study done by Amirsalari et al reported abnormal MRI findings in $28.5 \%$ of cases including cysts, tumors, white matter changes and brain atrophy. ${ }^{16}$

Where other studies have reported brain tumors comprising of $4 \%$ of all brain abnormalities presenting with seizures, our study has demonstrated brain tumors as the commonest brain abnormality comprising $10 \%$ of the studied population as 10 out of 100 patients demonstrated a brain tumor. ${ }^{17}$ 
Second most common finding was Periventricular leucomalacia $; 9 \%$ of study population. Brain atrophy is $6 \%$ was in accordance with a study done by Amirsalari et al which concluded it to be $10 \%$.

Brain abnormalities in our study also comprised of some developmental diseases like leucodystrophy (1\%), arachnoid cyst $1 \%$, agenesis of corpus callosum $(\mathrm{n}=1,1 \%)$. Similar study by Rachna Chaurasia and colleagues showed a different trend with leucodystrophy and cysts showing higher incidence $3.7 \%$ each. ${ }^{18}$ In that study, the infective pathologies were also reported to be occurring with different frequencies as compared to ours. A study conducted in developed countries, concluded cerebral dysgenesis as the most frequent cause of epilepsy(Hsieh DT and colleagues) ${ }^{19}$ Further studies by Guissard $\mathrm{G}$ and colleagues agreed with above findings and included, hypoxic-ischemic injury, non accidental injuries, infections, metabolic diseases and tumors as secondarily common cause for epilepsy. ${ }^{20}$ On the contrary, our study demonstrated congenital dysgenesis as third most common cause and brain tumors as the most common cause. Since MRI is more sensitive than CT with soft tissue imaging it is a technique of choice to image the focus of seizure origin (3 in Ravi Kumar). ${ }^{20}$

MRI is superior to CT even on contrast examination as majority of seizure activity originates from temporal lobes which are difficult to evaluate on CT due to the beam hardening artifact from underlying temporal bone. ${ }^{21}$ In literature, there are studies that support the relationship between abnormal brain MRI and continued seizure activity. ${ }^{22}$

MRI abnormality of brain was established to be prime independent modality to predict occurrence of convulsions in one epilepsy report. ${ }^{23} \mathrm{In}$ another report on partial seizures were seen to correlate with MRI detecting the underlying abnormality in $24 \%$ of cases. ${ }^{24}$ MRI and CT findings correlated in $14.8 \%$ of cases in a study done by Gelisse and colleagues on children with benign epilepsy with centrotemporal spikes. ${ }^{25} \mathrm{MRI}$ findings correlated in $38.6 \%$ of patients in a study conducted with Labate and co-workers on benign temporal epilepsy. ${ }^{26}$ Our data comprised of children presenting with first seizures referred to Bolan Medical Complex Quetta for MRI scan.

Our study was beneficial in evaluation of children presenting with epilepsy. Findings of MRI brain examination helped in fruitful decision making and better management of patients.

In the cases that were presented in our study, unremarkable MRI examinations were demarcated in $45 \%$ of cases may be due to inappropriate selection, stratification leading to erroneous referral to MRI of patients with pseudoseizures. This results in wastage of resources and malpractice as the already sick child is subjected to unnecessary anesthesia and turmoil of investigation.

\section{CONCLUSION:}

Brain magnetic resonance imaging was successful in detecting structural abnormalities and it can be trusted to detect seizure foci in pediatric patients.

\section{REFERENCES:}

1. Fisher RS, Acevedo C, Arzimanoglou A, Bogacz A, Cross JH, Elger CE, et al. ILAE official report: a practical clinical definition ofepilepsy. Epilepsia. 2014;55(4):475-482. doi: 10.1111/epi.12550.

2. Amirsalari S, Saburi A, Hadi R, Torkaman M, Beiraghdar F, Afsharpayman S, et al. Magnetic resonance imaging findings in epileptic children and its relation to clinical and demographic findings. Acta Med Iran. 2012;50(1):37-42.

3. Kwan P, Schachter SC, Brodie MJ. Drug-resistant epilepsy. N Engl J Med. 2011;365:919-926. doi: 10.1056/NEJMc 1111683.

4. Khatri ST, Iannaccone ST, Ilyas MS, Abdullah M, Saleem S. Epidemiology of Epilepsy in Pakistan: review of literature. J Pak Med Assoc 2003;53(12):594-7.

5. D.J. Pallin, J.N. Goldstein, J.S. Moussally, A.J. Pelletier, A.R. Green, C.A. Camargo Jr. Seizure visits in US emergency departments: epidemiology and potential disparities in care Int J Emerg Med, 2008;1(2): 97-105

6. Jackson GD, Briellmann RS, Kuzniecky RI. Temporal lobe Epilepsy. In: Kuzniecky RI, Jackson GD, editors. Magnetic Resonance in Epilepsy, 2005; 2: 99-220.

7. J.L. Martindale, J.N. Goldstein, D.J. Pallin Emergency department seizure epidemiology Emerg Med Clin North Am, 2011;29(1): 15-27

8. A.T. Berg, S.F. Berkovic, M.J. Brodie, J. Buchhalter, J.H. Cross, W. van Emde Boas, et al. Revised terminology and concepts for organization of seizures and epilepsies: report of the ILAE Commission on Classification and Terminology, 2005-2009 Epilepsia, 2010;51(4): 676-85

9. Malik MA, Akram RM, Tarar MA, Sultan A. Childhood epilepsy. J Coll Physician Surg Pak 2011;21(2):74-8.

10. C.L. Harden, J.S. Huff, T.H. Schwartz, R.M. Dubinsky, R.D. Zimmerman, S. Weinstein, et al. Reassessment: neuro-imaging in the emergency patient presenting with seizure (an evidencebased review): report of the Therapeutics and Technology Assessment Subcommittee of the American Academy of Neurology Neurology, 2007;69(18):1772-80

11. Commission on Neuroimaging, International League Against Epilepsy. Recommendations for neuroimaging of patients with epilepsy. Epilepsia. 1997;38:1255-56. doi: 10.1111/j.15281157.1997.tb01226.x.

12. Gilliam F, Wyllie E. Diagnostic testing of seizure disorders. Neurol Clin. 1996;14:61-84. doi:10.1016/S07338619(05)70243-7.

13. Kuzniecky RI. Neuroimaging in pediatric epilepsy. Epilepsia. 1996;37:10-21. doi: 10.1111/j.1528-1157.1996.tb06017.x.

14. Nordli DR, Pedley TA. Evaluation of children with seizures. In: Shinnar S, Amir N, Branski D, editors. Childhood Seizures, S. Karger, Basel; 1995. p. 67-77.

15. Bronen RA, Gupta V. Epilepsy. In: Atlas SW editor. Magnetic Resonance Imaging of Brain and Spine. 4th ed, 2009. p.307-42. 
16. Resta M, Palma M, Dicuonzo F, Spagnolo P, Specchio LM, Laneve A, et al. Imaging studies in partial epilepsy in children and adolescents. Epilepsia 1994;35(6):1187-93.

17. Amirsalari S, Saburi A, Hadi R, Torkaman M, Beiraghdar F, Afsharpayman $\mathrm{S}$, et al. Magnetic resonance imaging findings in epileptic children and its relation to clinical and demographic findings. Acta Med Iran 2012;50(1):37-42.

18. Chaurasia R, Singh S, Mahur S, Sachan P. Imaging in pediatric epilepsy: spectrum of abnormalities detected on MRI. J Evol Med Dent Sci 2013;19(2):3377-87.

19. Hsieh DT, Chang T, Tsuchida TN, Vezina LG, Vanderver A, Siedel J, et al. New-onset afebrile seizures in infants: role of Neuroimaging. Neurology 2010;74(2):150-6.

20. Guissard G, Damry N, Dan B, David P, Sekhara T, Ziereisen $\mathrm{F}$, et al. Imaging in pediatric epilepsy. Arch Pediatr 2005; 12(3):337-46.

21. Theodore WH, Dorwart R, Holmes M, Porter RJ, DiChiro G. Neuroimaging in refractory partial seizures - comparison of PET, CT and MRI. Neurology 1986;36(6):750-9.

22. Spooner CG, Berkovic SF, Mitchell LA, Wrennall JA, Harvey AS. New-onset temporal lobe epilepsy in children: lesion on
MRI predicts poor seizure outcome. Neurology 2006;67(12):2147-53.

23. Gaillard WD, Weinstein S, Conry J, Pearl PL, Fazilat S, Fazilat S, et al. Prognosis of children with partial epilepsy: MRI and serial 18FDG-PET. Neurology 2007;68(9):655-9.

24. Betting LE, Mory SB, Lopes-Cendes I, Li LM, Guerreiro MM, Guerreiro CA, et al. MRI reveals structural abnormalities in patients with idiopathic generalized epilepsy. Neurology 2006;67(5):848-52.

25. Gelisse P, Corda D, Raybaud C, Dravet C, Bureau M, Genton $\mathrm{P}$. Abnormal neuroimaging in patients with benign epilepsy with centrotemporal spikes. Epilepsia 2003;44(3):372-8.

26. Labate A, Ventura P, Gambardella A, Le Piane E, Colosimo $\mathrm{E}$, Leggio U, et al. MRI evidence of mesial temporal sclerosis in sporadic "benign" temporal lobe epilepsy. Neurology 2006;66(4):562-5. 